\title{
Prediction of Materials Density according to Number of Scattered Gamma Photons Using Optimum Artificial Neural Network
}

\author{
Gholam Hossein Roshani, ${ }_{1}^{1}$ Seyed Amir Hossein Feghhi, ${ }^{1}$ Farzin Shama, ${ }^{2,3}$ \\ Abolfazl Salehizadeh, ${ }^{4}$ and Ehsan Nazemi ${ }^{1}$ \\ ${ }^{1}$ Radiation Application Department, Shahid Beheshti University, G.C., Tehran, Iran \\ ${ }^{2}$ Electrical Engineering Department, Razi University, Kermanshah, Iran \\ ${ }^{3}$ Electrical Engineering Department, Islamic Azad University of Kermanshah, Kermanshah, Iran \\ ${ }^{4}$ Nuclear Engineering Department, Amirkabir University of Technology, Tehran, Iran \\ Correspondence should be addressed to Gholam Hossein Roshani; hosseinroshani@yahoo.com
}

Received 26 January 2014; Revised 22 April 2014; Accepted 22 April 2014; Published 10 June 2014

Academic Editor: Sebastien Incerti

Copyright (c) 2014 Gholam Hossein Roshani et al. This is an open access article distributed under the Creative Commons Attribution License, which permits unrestricted use, distribution, and reproduction in any medium, provided the original work is properly cited.

\begin{abstract}
Through the study of scattered gamma beam intensity, material density could be obtained. Most important factor in this densitometry method is determining a relation between recorded intensity by detector and target material density. Such situation needs many experiments over materials with different densities. In this paper, using two different artificial neural networks, intensity of scattered gamma is obtained for whole densities. Mean relative error percentage for test data using best method is $1.27 \%$ that shows good agreement between the proposed artificial neural network model and experimental results.
\end{abstract}

\section{Introduction}

The gamma-ray photons lose their energy in a stopping medium by these processes: photoelectric effect, Compton effect, pair production, and photonuclear effect. With the analysis of these interactions, some information about characteristics of materials can be obtained. Compton scattering is strongly dependent on the materials density. Therefore, this method is very good choice for densitometry of unknown materials [1-6]. El Abd [7] has shown that scattering photons are more sensitive than transmitted photons in order to density meter and void fraction prediction. In [7], the void fraction has been predicted without using artificial neural network (ANN); therefore the error is considerable. In this paper, experimental data have been obtained from a density measurement tomography system [8]. These data were used for training the ANN. Set-up of this tomography system was shown in Figure 1.

In investigated tomography system [8], a $\mathrm{NaI}(\mathrm{Tl})$ scintillation detector in $3 \times 3$ inch dimensions has been used.
The detector records scattered photons from target sample. The source is ${ }^{137} \mathrm{Cs}$ with $8 \mathrm{mci}$ activation. Distances between the sample from the source collimator and the detector are $5.56 \mathrm{~cm}$ and $6 \mathrm{~cm}$, respectively. Investigated samples have dimensions and thickness of $1 \times 2 \mathrm{~cm}^{2}$ and $1 \mathrm{~cm}$, respectively. The time durations of measurement are 100 seconds. Relative stochastic error has inverse relation with the number of registered counts in detector. In this study because the scattering method is used and the number of counts in this method is less in comparison with the transmission method, therefore the measurement time should be increased in order to decrease the stochastic error. By taking 100second time duration, the stochastic errors are in the range of $0.5 \%-1.5 \%$ (5000-20000 counts for different materials). The experimental results are shown in Table 1.

The aim of this study is increasing the precision of described tomography system by means of artificial neural networks (ANNs). In [9-11], some applications of artificial intelligence in density prediction and volume fraction 


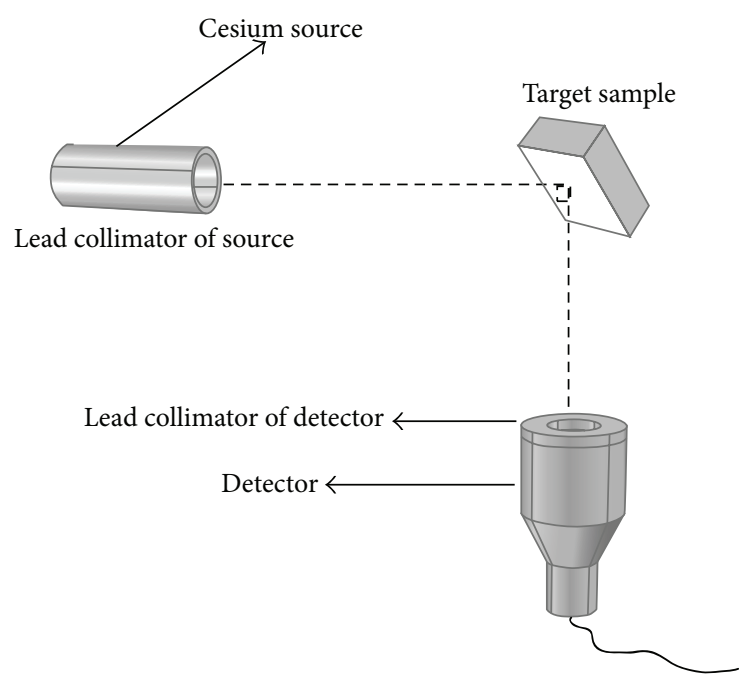

FIGURE 1: Schematic of tomography structure system.

TABLE 1: Number of detected photons from different materials.

\begin{tabular}{lcc}
\hline Tested materials & $\begin{array}{c}\text { Number of detected } \\
\text { photons }(\#)\end{array}$ & $\begin{array}{c}\text { Density of material } \\
\left(\mathrm{g} / \mathrm{cm}^{3}\right)\end{array}$ \\
\hline Polyethylene & 5212 & 0.91 \\
Calcium & 7301 & 1.54 \\
Magnesium & 8125 & 1.73 \\
Beryllium & 9094 & 1.86 \\
Sulfur & 9631 & 2.06 \\
Glass & 11307 & 2.5 \\
Aluminum & 11705 & 2.7 \\
Titanium & 16185 & 4.54 \\
Zinc & 19701 & 7.13 \\
Iron & 20429 & 7.87 \\
\hline
\end{tabular}

measurement have been presented. In this study, registered counts in the scattering detector, which are obtained for some known materials with various densities by the experiments, are used as the input of the ANN. Also, the densities are used as the output of the ANN. In such situation, with one input and one output, increasing the hidden layers not only decreases error but also can increase it. Hence, two optimum structures with low error for using in densitometry system are proposed. Finally, using these feedforward multilayer perceptron ANNs, numbers of detected photons were obtained for some unknown materials.

\section{First Suggested ANN}

ANNs are so useful in prediction problems. ANNs can be defined as a mathematical system consisting of simple processing elements named neuron running in parallel, which can be generated as one or multiple layers [12]. The multilayer perceptron (MLP) networks are the most widely used neural networks that are feedforward networks with minimum three layers (input layer, hidden layer, and output layer). Each layer has a number of processing units and each unit is fully interconnected with weighted connections to units in
TABLE 2: Specification of first proposed ANN model.

\begin{tabular}{lc}
\hline Neural network & MLP \\
\hline Number of neurons in the input layer & 1 \\
Number of neurons in the first hidden layer & 5 \\
Number of neurons in the second hidden layer & 4 \\
Number of neurons in the output layer & 1 \\
Number of epochs & 200 \\
Activation function & tansig \\
\hline
\end{tabular}

the subsequent layer [13]. In this paper, two precise methods based on multilayer perceptron ANN have been presented to predict material density according to counted scattered photons. The first proposed MLP model is shown in Figure 2. The input variable used in this model is number of counted photons and the output is material density. The input to the node $l$ in the first hidden layer is given by $[12,13]$ :

$$
\eta_{l}=\sum_{u=1}^{1}\left(X_{u} w_{u l}\right)+\theta_{l}, \quad l=1,2, \ldots, 5 .
$$

The output from $l$ th neuron of the second hidden layer is given by

$$
O_{l}=f\left(\eta_{l}\right)
$$

where $X$ is the input variables, $\theta$ is the bias term, $w$ is the weighting factor, and $f$ is the activation function of the hidden layer.

Required data set for training the network is obtained from described tomography system in the introduction. The training of presented MLP networks is done by LevenbergMarquardt (LM) algorithm. In this method, first derivative and second derivative (hessian) are used for network weight correction [14]. One of the advantages of LM method is that it is not necessary to determine the learning rate from the beginning. The learning rate in this method can be updated adaptively. In order to develop the ANN model the total data are divided into two classes: $70 \%$ for training and 30\% for testing the trained model. MATLAB 7.6.0 software was used for training the ANN model. Table 2 shows the specification of the first proposed ANN model being used in this study.

Table 3 shows the obtained results for the proposed ANN model, where the mean relative error percentage (MRE\%), the root mean square error (RMSE), and the mean absolute error percentage (MAE\%) of the network are calculated by

$$
\begin{gathered}
\text { MRE } \%=100 \times \frac{1}{N} \sum_{i=1}^{N}\left|\frac{X_{i}(\text { Exp })-X_{i}(\text { Pred })}{X_{i}(\text { Exp })}\right|, \\
\text { RMSE }=\left[\frac{\sum_{i=1}^{N}\left(X_{i}(\text { Exp })-X_{i}(\text { Pred })\right)^{2}}{N}\right]^{0.5}, \\
\text { MAE } \%=100 \times \frac{1}{N} \sum_{i=1}^{N} \mid X_{i}(\text { Exp })-X_{i}(\text { Pred }) \mid
\end{gathered}
$$

where $N$ is the number of data and " $X(\operatorname{Exp})$ " and " $X$ (Pred)" stand for experimental and predicted (ANN) values, respectively. 


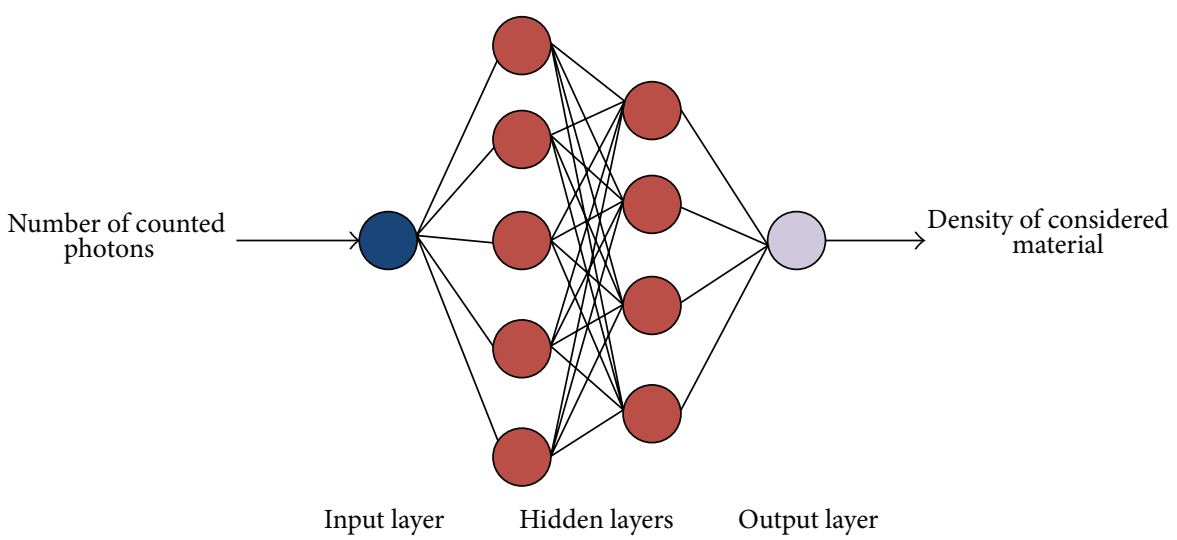

Figure 2: Proposed architecture for the first MLP model.

TABLE 3: Obtained errors for training and testing results of the first proposed ANN model.

\begin{tabular}{lcc}
\hline Error & Train & Test \\
\hline MRE\% & 1.1103 & 1.2763 \\
MAE\% & 2.2316 & 2.9692 \\
RMSE & 0.0288 & 0.0321
\end{tabular}

The training and testing results of the presented ANN model are shown in Figure 3. From Table 3 and Figure 3, clearly the predicted unknown material density by ANN model is close to the experimental results. These results show the applicability of ANN as an accurate and reliable model for the prediction of density according to the counted gamma photons.

Prediction results about number of the scattered photons from some different materials are given in Table 4.

\section{Second Suggested ANN}

To have simpler and smaller size network, by considering the number and variety of the inputs, another network is proposed, as shown in Figure 5. The proposed ANN includes only two layers and two neurons; each layer consists of one neuron.

The properties of the second suggested ANN are illustrated in Table 5. As shown in this Table, the presented network has smaller and more optimum size in comparison with the first suggested ANN. Furthermore, the number of epochs for weight iterations to approach the requested response is increased. LM learning results in local convergences of the gradient function in primal weight corrections. To avoid this fact, the epoch of the iterations is increased. Due to second order convergence of LM learning, the learning process has a good speed and it can achieve the requested response, rapidly.

Like the previous network, whole of the data are parted in two classes: $70 \%$ for train and 30\% for test. The defined errors for training and testing of the second proposed ANN are shown in Table 6.

As seen from Table 6, the MAE\% and MRE\% of this network architecture for train and test data and also the RMSE error for train data have significant values compared
TABle 4: Prediction of materials according to number of detected photons using first ANN.

\begin{tabular}{lcc}
\hline $\begin{array}{l}\text { Prediction of } \\
\text { materials }\end{array}$ & $\begin{array}{c}\text { Number of detected } \\
\text { photons }(\#)\end{array}$ & $\begin{array}{c}\text { Density of material } \\
\left(\mathrm{g} / \mathrm{cm}^{3}\right)\end{array}$ \\
\hline Water & 5478 & 1 \\
Sulfuric acid & 8778 & 1.840 \\
Bone & 8811 & 1.85 \\
Salt & 10190 & 2.200 \\
Carbon & 11512 & 2.60 \\
Granite & 11629 & 2.650 \\
Sand & 12080 & 2.800 \\
Tin & 19867 & 7.30 \\
Brass & 20944 & 8.40 \\
Nickel & 21316 & 8.80 \\
Silver & 22865 & 10.40 \\
Lead & 23794 & 11.30 \\
\hline
\end{tabular}

TABle 5: Specification of second proposed ANN model.

\begin{tabular}{lc}
\hline Neural network & MLP \\
\hline Number of neurons in the input layer & 1 \\
Number of neurons in the first hidden layer & 1 \\
Number of neurons in the second hidden layer & 1 \\
Number of neurons in the output layer & 1 \\
Number of epochs & 400 \\
Activation function & tansig \\
\hline
\end{tabular}

TABLE 6: Obtained errors for training and testing results of the second proposed ANN model.

\begin{tabular}{lcc}
\hline Error & Train & Test \\
\hline MRE\% & 2.1283 & 3.8018 \\
MAE\% & 2.9861 & 4.6482 \\
RMSE & 0.0787 & 0.0167 \\
\hline
\end{tabular}

to the pervious complex network. But, the value of RMSE error for test data has a small amount of 0.0167 , which is the most important factor. The RMSE has $192.21 \%$ reduction from the previous architecture and validates the efficiency of this simple and optimum network. 


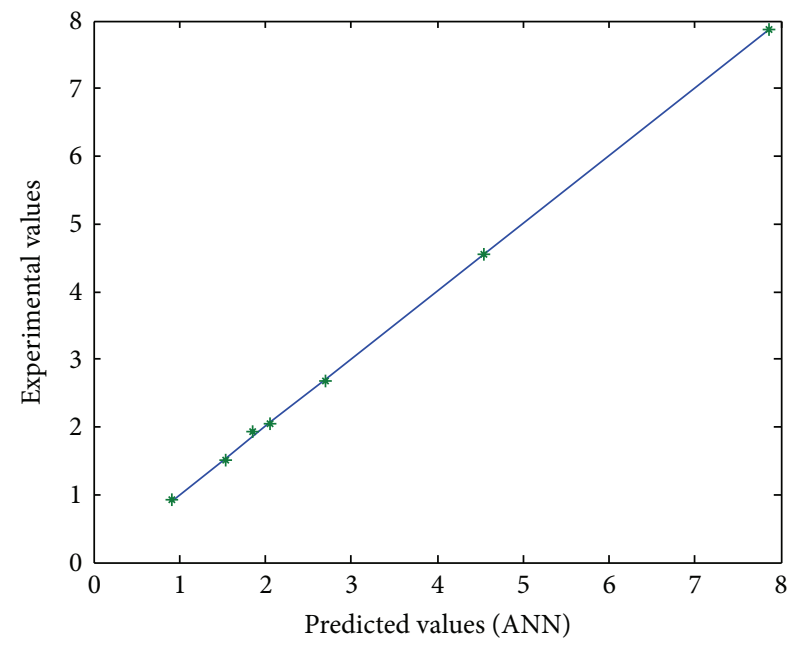

(a)

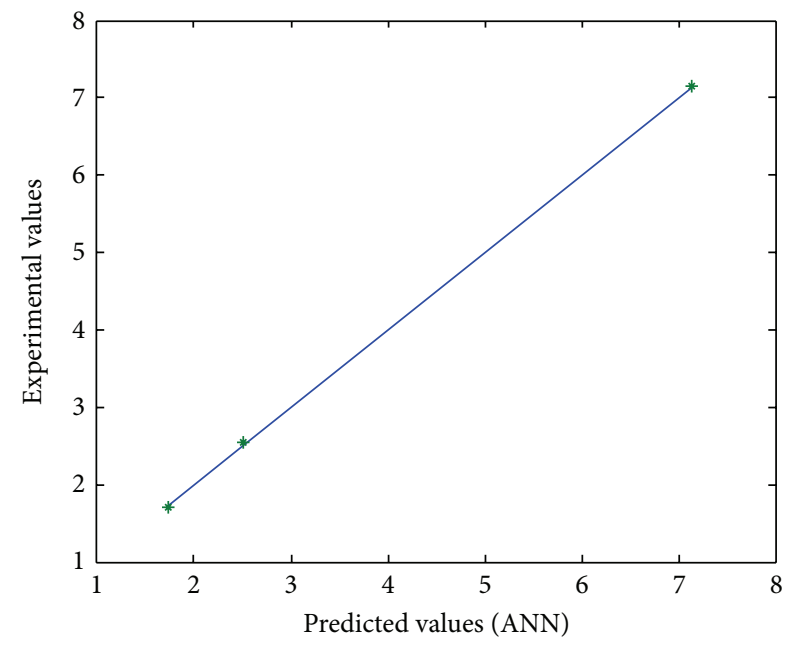

(b)

FIgURE 3: Comparison of experimental and predicted (first ANN model) results for (a) training data and (b) testing data.

Train and test results of the proposed ANN model are shown in Figure 5. The simulation results are in good agreement with experimental result in train and test mode.

Most important advantage of this architecture, in comparison with the previous one, is avoidance of the complications, which simplifies the implementation of the proposed ANN. In addition, a simple relation can be determined from this network to calculate the density of materials as a function of number of detected photons:

$$
\begin{aligned}
D= & \frac{75.3974}{1+\exp ((3.248 \exp (M)-55.8484) /(1+\exp (M)))} \\
& -3.8825\left(\mathrm{gr} / \mathrm{cm}^{3}\right),
\end{aligned}
$$

where $M=-6.506 \times 10^{-5} \mathrm{~N}+4.9388, N=$ number of detected photons, and $D=$ density of material $\left(\mathrm{gr} / \mathrm{cm}^{3}\right)$.

It is for the first time that a simple formula is presented, which can predict the density of every material according to the scattered counted photons with high precision.

The predicted results about applied materials in Table 4 using second ANN are given in Table 7.

\section{ANN Validation Using $K$-Fold Cross Validation}

For a neural network, which has a few number of inputs, the validation is so necessary to ensure the achieved results and predictions. Several methods have been presented for validation such as $K$-fold cross validation [15]. In this method, the data are parted to $K$ batches. The value of $K$ can be determined by the requested accuracy and the numbers of inputs. Firstly, one of these batches is considered as test inputs, while the others are assumed as train inputs. Then the considered batch as test inputs will be classified in train
TABLe 7: Prediction of materials according to number of detected photons using second proposed ANN model.

\begin{tabular}{lcc}
\hline $\begin{array}{l}\text { Prediction of } \\
\text { materials }\end{array}$ & $\begin{array}{c}\text { Number of detected } \\
\text { photons }(\#)\end{array}$ & $\begin{array}{c}\text { Density of material } \\
\left(\mathrm{g} / \mathrm{cm}^{3}\right)\end{array}$ \\
\hline Water & 5003 & 1 \\
Sulfuric acid & 8994 & 1.840 \\
Bone & 9035 & 1.85 \\
Salt & 10310 & 2.200 \\
Carbon & 11595 & 2.60 \\
Granite & 11738 & 2.650 \\
Sand & 12179 & 2.800 \\
Tin & 19854 & 7.30 \\
Brass & 20972 & 8.40 \\
Nickel & 21351 & 8.80 \\
Silver & 22684 & 10.40 \\
Lead & 23348 & 11.30 \\
\hline
\end{tabular}

inputs and another batch from the train inputs will be applied to the network. The above described trend is continuing $K$ time. Finally, test errors for all of the occurred training states are calculated. The error averages are compared with test errors of the proposed ANN and can validate the accuracy of the modeled ANN for next results and predictions. For the proposed method $K$ is considered five and data are classified as twin batches: [5212,20429] [7301, 19701] [8152, 16185] [9094, 11705] [9631, 11307]. Because of using LM learning, the epoch is fixed to 400 for all of the training processes. The validation results show that the second suggested ANN has less error average of RMSE in various tests (0.0934) (Figure 4). This value is an acceptable validation average error for a MLP in weight correction iterations. The variation of RMSE errors for various batches in order to various $K s$ is illustrated in Figure 6. 




FIGURE 4: Proposed model of the second suggested ANN with two perceptron hidden layers.

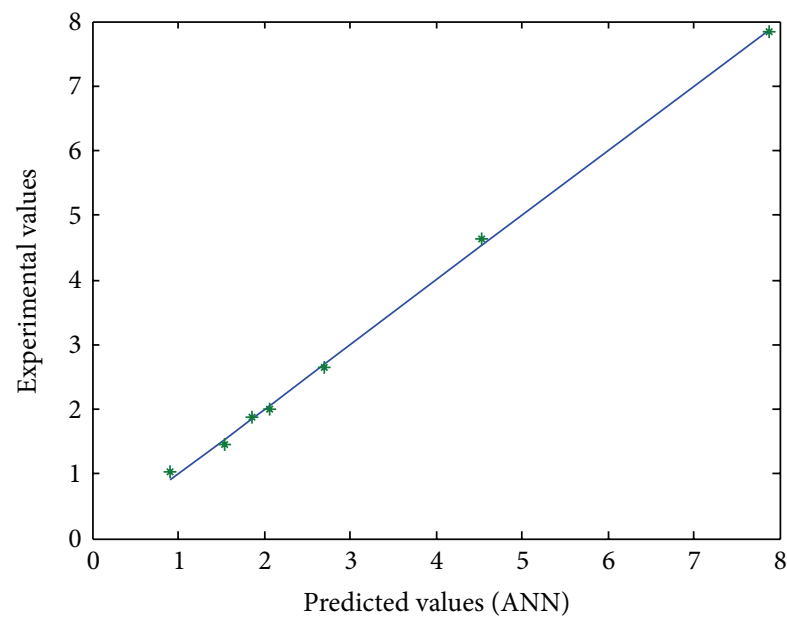

(a)

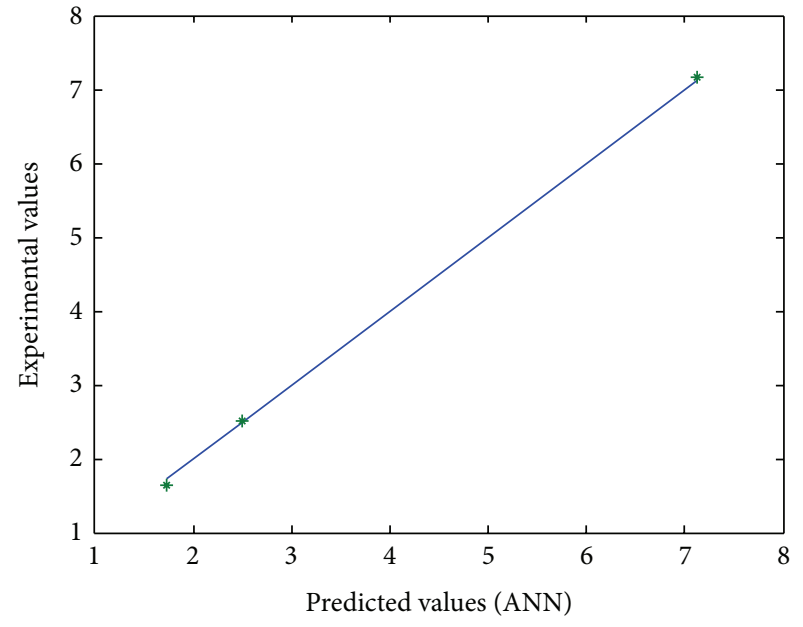

(b)

FIGURE 5: Comparison of experimental and predicted (second ANN model) results for (a) training data and (b) testing data.



FIGURE 6: The variation of RMSE errors for various batches in order to various $K$ s.

\section{Conclusion}

The number of scattered and counted gamma photons highly depends on material density. A tomography system based on this principle was designed in order to measure the density of various materials. The measurement precision in such systems is an important issue in the industry. One solution for improving the precision is increasing the number of experiments and calibration measurements with more and more different materials, which is a hard task due to problems related to experimental works. Another solution is using the artificial intelligence which is an easy and powerful tool in prediction problems. In this study, registered counts in the scattering detector obtained by the designed tomography system are used as the input of the ANN. Also the densities are used as the output of the ANN. Two different multilayer perceptron artificial neural networks are proposed to predict material density. According to few numbers of inputs and having one kind of input and output, $K$-fold cross validation was applied. The results of proposed ANNs show that the presented model could be employed in densitometry of materials. Using second ANN, a simple formula to predict the materials density was presented.

\section{Conflict of Interests}

The authors declare that there is no conflict of interests regarding the publication of this paper.

\section{References}

[1] G. Schlieper, "Applying gamma ray densitometry in a PM production plant," Metal Powder Report, vol. 56, no. 1, pp. 22-27, 2001.

[2] E. Åbro, V. A. Khoryakov, G. A. Johansen, and L. Kocbach, "Determination of void fraction and flow regime using a neural network trained on simulated data based on gamma-ray densitometry," Measurement Science and Technology, vol. 10, no. 7, pp. 619-630, 1999.

[3] S. A. Tjugum, B. T. Hjertake, and G. A. Johansen, "Multiphase flow regime identification by multibeam gamma-ray densitometry," Measurement Science and Technology, vol. 13, no. 8, pp. 1319-1326, 2002.

[4] S.-A. Tjugum, J. Frieling, and G. A. Johansen, "A compact low energy multibeam gamma-ray densitometer for pipe-flow 
measurements," Nuclear Instruments and Methods in Physics Research B: Beam Interactions with Materials and Atoms, vol. 197, no. 3-4, pp. 301-309, 2002.

[5] C. M. Salgado, C. M. N. A. Pereira, R. Schirru, and L. E. B. Brandão, "Flow regime identification and volume fraction prediction in multiphase flows by means of gamma-ray attenuation and artificial neural networks," Progress in Nuclear Energy, vol. 52, no. 6, pp. 555-562, 2010.

[6] M. Khorsandi and S. A. H. Feghhi, "Design and construction of a prototype gamma-ray densitometer for petroleum products monitoring applications," Measurement, vol. 44, no. 9, pp. 1512$1515,2011$.

[7] A. El Abd, "Intercomparison of gamma ray scattering and transmission techniques for gas volume fraction measurements in two phase pipe flow," Nuclear Instruments and Methods in Physics Research A: Accelerators, Spectrometers, Detectors and Associated Equipment, vol. 735, pp. 260-266, 2014.

[8] S. Ashrafi, D. Alizadeh, and O. Jahanbakhsh, "Application of artificial neural networks in densitometry using compton scattering," in Proceedings of the Iran Physics Conference, 2012.

[9] G. H. Roshani, S. A. H. Feghhi, A. Mahmoudi-Aznaveh, E. Nazemi, and A. Adineh-Vand, "Precise volume fraction prediction in oil-water-gas multiphase flows by means of gamma-ray attenuation and artificial neural networks using one detector," Measurement, vol. 51, pp. 34-41, 2014.

[10] G. H. Roshani, S. A. H. Feghhi, A. Adineh-Vand, and M. Khorsandi, "Application of adaptive neuro-fuzzy inference system in prediction of fluid density for a gamma ray densitometer in petroleum products monitoring," Measurement, vol. 46, no. 9, pp. 3276-3281, 2013.

[11] M. Khorsandi, S. A. H. Feghhi, A. Salehizadeh, and G. H. Roshani, "Developing a gamma ray fluid densitometer in petroleum products monitoring applications using Artificial Neural Network," Radiation Measurements, vol. 59, pp. 183-187, 2013.

[12] J. G. . Taylor, Neural Networks and Their Applications, John Wiley \& Sons, West Sussex, UK, 1996.

[13] A. R. Gallant and H. White, "On learning the derivatives of an unknown mapping with multilayer feedforward networks," Neural Networks, vol. 5, no. 1, pp. 129-138, 1992.

[14] M. T. Hagan and M. B. Menhaj, "Training feedforward networks with the Marquardt algorithm," IEEE Transactions on Neural Networks, vol. 5, no. 6, pp. 989-993, 1994.

[15] J. D. Rodriguez, A. Perez, and J. A. Lozano, "Sensitivity analysis of k-fold cross validation in prediction error estimation," IEEE Transactions on Pattern Analysis and Machine Intelligence, vol. 32, no. 3, pp. 569-575, 2010. 

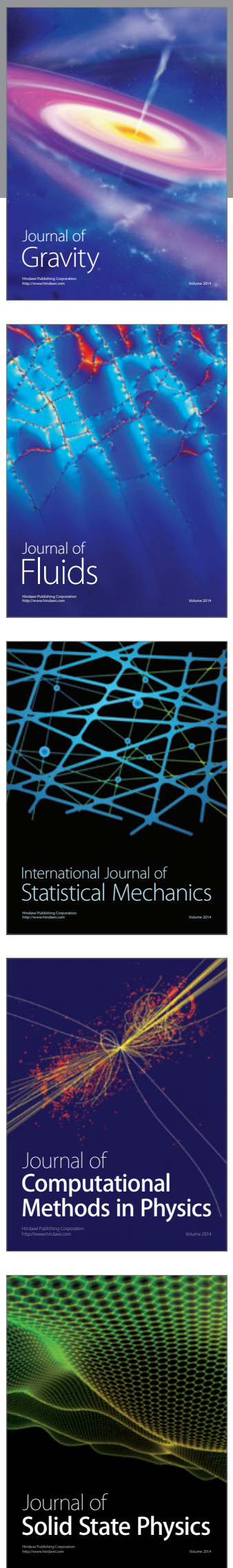

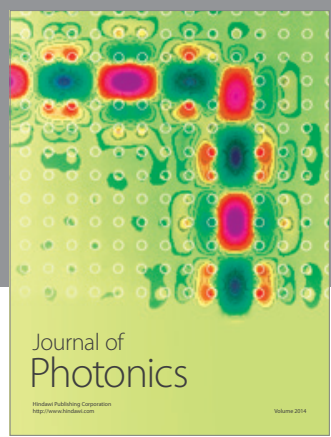

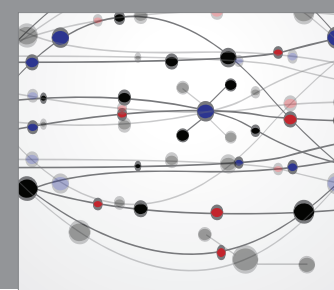

The Scientific World Journal

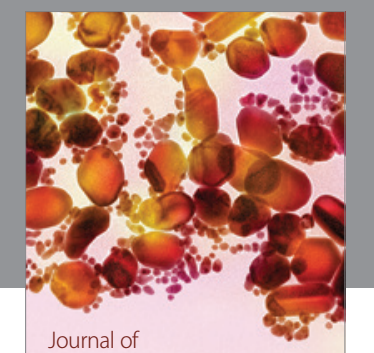

Soft Matter
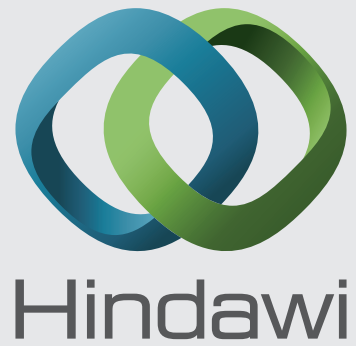

Submit your manuscripts at

http://www.hindawi.com
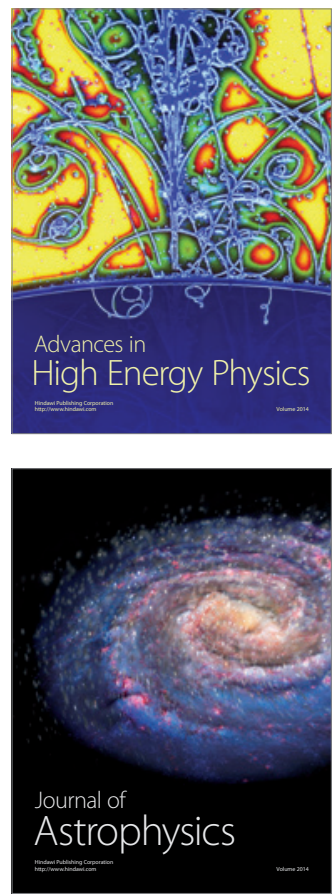
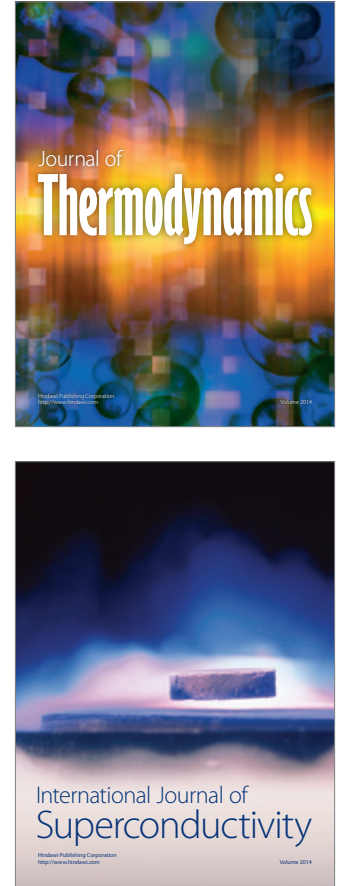
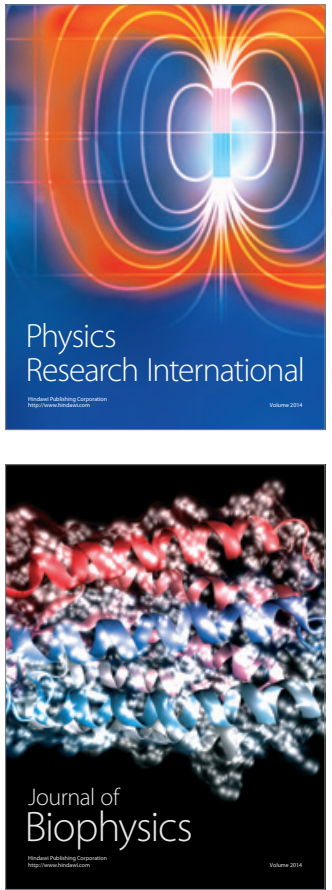
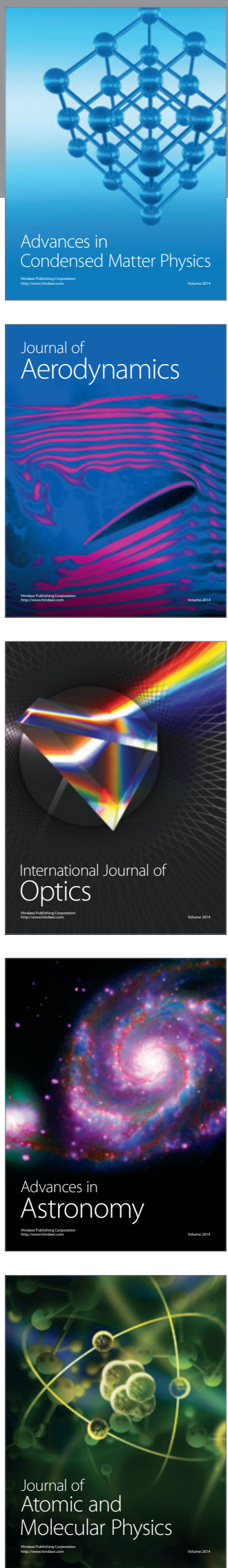